\title{
Cryptococcal osteomyelitis of the Zygomatic bone: a case report
}

\author{
Takashi Matsuki* ${ }^{*}$, Shunsuke Miyamoto and Taku Yamashita
}

\begin{abstract}
Background: Disseminated cryptococcosis is a well-characterized complication in immunocompromised patients with cryptococcal pneumonia or meningitis; however, isolated cryptococcal osteomyelitis is a rare entity that occurs in approximately $5 \%$ of patients with cryptococcosis. Cryptococcal osteomyelitis in the head and neck region is extremely rare. To the best of our knowledge, no cases of cryptococcal osteomyelitis affecting only the zygomatic bone have been reported to date.

Case presentation: A 78-year-old man without other comorbidities presented with progressive swelling of the right cheek along with pain and trismus. Clinical examination revealed a tender swelling in the right zygomatic region; the maximal mandibular opening was about $2 \mathrm{~cm}$. Laboratory data showed mildly elevated inflammatory indices (C-reactive protein: $0.45 \mathrm{mg} / \mathrm{dL}$; erythrocyte sedimentation rate: $35 \mathrm{~mm} / \mathrm{h}$ ). Computed tomography showed a $30-\mathrm{mm}$-diameter lesion at the right zygomatic arch. A part of the lesion has extended to the subcutaneous area of the cheeks with signs of bone destruction and surrounding contrast effects. Histopathological examination of fine-needle aspirate and needle biopsy showed cryptococcus. Furthermore, culture of the aspirate showed growth of Cryptococcus neoformans. No evidence of any other site involvement was observed. Therefore, the patient was diagnosed with isolated cryptococcal osteomyelitis and was initiated on fluconazole therapy. The treatment was effective, and all symptoms were resolved in 4 weeks. Fluconazole therapy was stopped after 6 months. There are no signs of recurrence as of 15-month follow-up. The patient has no cosmetic abnormalities or sequelae.
\end{abstract}

Conclusions: Fine-needle aspiration cytology, needle biopsy, and fungal culture were useful for definitive diagnosis. Immunocompetent patients with isolated osteomyelitis may be cured with oral fluconazole alone.

Keywords: Cryptococcosis, Osteomyelitis, Zygomatic bone, Fluconazole, Case report

\section{Background}

Cryptococcosis is an opportunistic infection that typically occurs in immunocompromised hosts such as patients with acquired immunodeficiency syndrome, hematologic malignancy, hepatic failure, and sarcoidosis, who underwent stem cell or solid organ transplant, or those on long-term steroid therapy. Disseminated cryptococcosis is a well-known complication of cryptococcal pneumonia or meningitis [1]. However, isolated

\footnotetext{
*Correspondence: matsuki@med.kitasato-u.ac.jp

Department of Otorhinolaryngology, Head and Neck Surgery, Kitasato University School of Medicine, 1-15-1 Kitasato, Minami-ku, Sagamihara, Kanagawa 252-0374, Japan
}

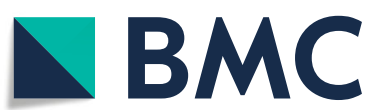

C C The Author(s). 2020 Open Access This article is licensed under a Creative Commons Attribution 4.0 International License, which permits use, sharing, adaptation, distribution and reproduction in any medium or format, as long as you give appropriate credit to the original author(s) and the source, provide a link to the Creative Commons licence, and indicate if changes were made. The images or other third party material in this article are included in the article's Creative Commons licence, unless indicated otherwise in a credit line to the material. If material is not included in the article's Creative Commons licence and your intended use is not permitted by statutory regulation or exceeds the permitted use, you will need to obtain permission directly from the copyright holder. To view a copy of this licence, visit http://creativecommons.org/licenses/by/4.0/ The Creative Commons Public Domain Dedication waiver (http://creativecommons.org/publicdomain/zero/1.0/) applies to the data made available in this article, unless otherwise stated in a credit line to the data.

cryptococcal osteomyelitis is a rare entity that accounts for approximately $5 \%$ of patients with cryptococcosis [2-4]. A systematic review revealed that only 89 cases of cryptococcosis are documented in the published literature [5, 6]. Cryptococcal osteomyelitis is usually caused by hematogenous spread from a primary lung infection after inhalation of microscopic airborne fungal spores $[5,6]$. Direct inoculation of fungal spores due to trauma is another potential route of infection [5, 7]. Most reported cases of osseous cryptococcosis were due to Cryptococcus neoformans $[3,5]$. Although the condition typically occurs in immunocompromised patients secondary to disseminated 
cryptococcosis, isolated osteomyelitis may occur in immunocompetent patients who have no apparent underlying disease or immune deficiency $[1,4,8]$. Systemic signs such as fever or fatigue are often not observed [3]. The most commonly reported sites of infection are the vertebrae, skull, and femur, respectively [5]. Infection of the zygomatic bone has been reported as a rare complication of otogenic infection such as otitis media or mastoiditis [9, 10]. Cryptococcal osteomyelitis in the head and neck region is extremely rare, and osteomyelitis of zygomatic bone alone has never been reported.

Here, we present the first documented case of cryptococcal isolated osteomyelitis of the zygomatic bone in an immunocompetent patient. Written informed consent of the patient has been obtained for publication of this case report and the accompanying images.

\section{Case presentation}

A 78-year-old man presented with a 2-week-long history of increasing right cheek swelling, pain, and trismus. He had no history of cheek trauma or fever. He had a history of prostate cancer that was treated with radiation therapy 2 years ago. He was not on any immunosuppressive treatment and did not experience previous recurrent or severe infections. He has no relevant exposures for Cryptococcus, such as that from birds or soil. There was no history of smoking or alcohol consumption. Clinical examination revealed swelling of the right zygomatic region (Fig. 1) with severe spontaneous pain and tenderness. His maximal mandibular opening was about $2 \mathrm{~cm}$. On laboratory investigations, his serum C-reactive protein level was $0.45 \mathrm{mg} / \mathrm{dL}$ (reference range, $0-0.14$ ), total white blood cell count was $6700 / \mathrm{mm}^{3}$ (reference range, 3300-8600) with $54.4 \%$ neutrophils, and erythrocyte sedimentation rate (ESR) was $35 \mathrm{~mm} / \mathrm{h}$ (reference range, 2-10). Serum levels of alkaline phosphatase, calcium, phosphorous, glucose, and glycosylated hemoglobin A1c were within the normal range. His serum alanine aminotransferase was $11 \mathrm{IU} / \mathrm{L}$ (reference range, 10-42) and aspartate transaminase level was $22 \mathrm{IU} / \mathrm{L}$ (reference range, 13-30). The viral serology tests for hepatitis $B$ and hepatitis $\mathrm{C}$ yielded negative results. Contrastenhanced computed tomography (CT) scan showed the lesion in the right zygomatic arch with a maximum diameter of $30 \mathrm{~mm}$. There were signs of bone destruction and surrounding contrast effects (Fig. 2). The lesion had extended to the subcutaneous tissue in the cheek; however, there were no signs of hardening or calcification. These findings are suggestive of a malignant tumor derived from the zygomatic bone or a focal abscess with features not typical of an infection caused by common mechanisms such as by extension of an otogenic infection. Cryptococcus was found in the fine-needle

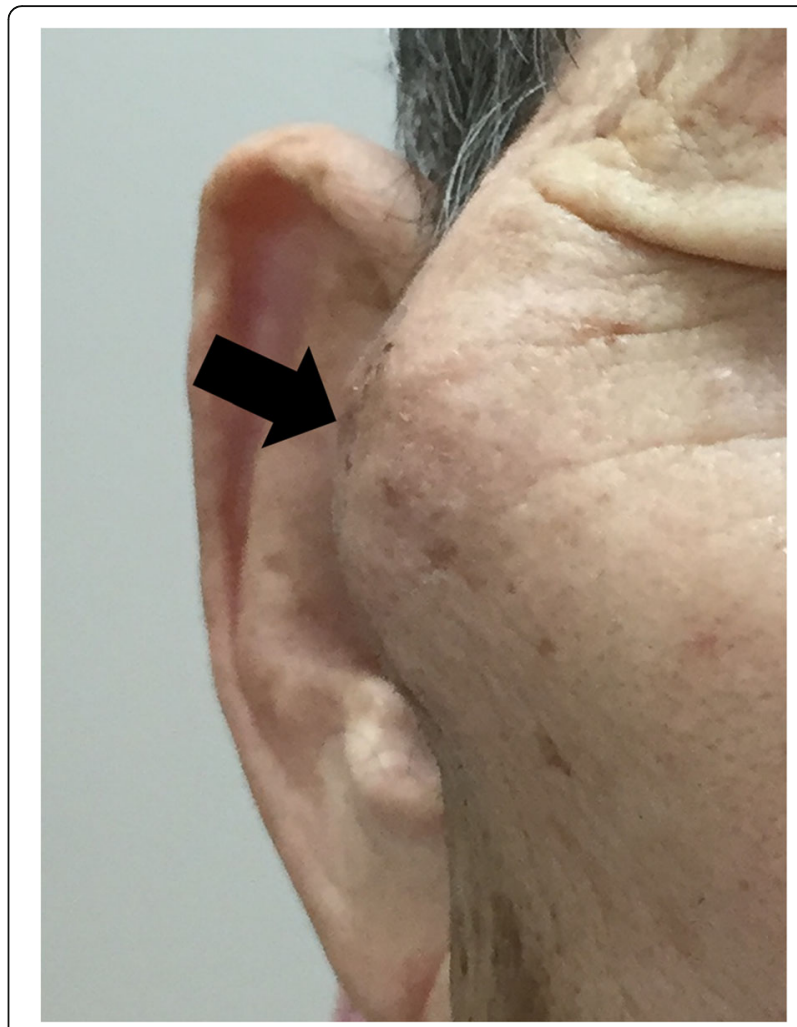

Fig. 1 Photograph of the patient prior to initiation of treatment. The right zygomatic region shows swelling with erythema (arrow)

aspiration cytology (FNAC) based on Grocott staining; additional histopathological diagnosis of needle biopsy from the mass was non-necrotizing granuloma with Cryptococcus (Fig. 3). Furthermore, culture of the aspirate showed growth of Cryptococcus neoformans, and the minimal inhibitory concentration of fluconazole for cryptococcus was low at $2.0 \mu \mathrm{g} / \mathrm{ml}$. Tests for human immunodeficiency virus (HIV) antigens and antibodies were negative. Lumbar puncture (LP) revealed clear and colorless cerebrospinal fluid (CSF) with an opening pressure of $70 \mathrm{mmH}_{2} \mathrm{O}$. The CSF cell count was $<1 / \mu \mathrm{L}$ (reference range, 0-5), and glucose and protein levels were within normal limits $[67 \mathrm{mg} / \mathrm{dL}$ (reference range, 50-75) and $34 \mathrm{mg} / \mathrm{dL}$ (reference range, 10-40), respectively]. The CSF tested negative for cryptococcal antigens. CSF and blood cultures showed no bacterial or fungal growth. Brain magnetic resonance imaging (MRI), and chest CT. Finally, the patient was diagnosed with isolated cryptococcal osteomyelitis of the zygomatic bone and initiated on fluconazole therapy ( $400 \mathrm{mg}$ daily). By 2 weeks, the swelling remarkably improved with alleviation of both trismus and pain. By 4 weeks, the swelling had disappeared (Fig. 4). Fluconazole therapy was stopped after 6 months. Follow-up examination at 15 months showed a very small palpable depression in the zygomatic area; however, there were no signs of lesion 

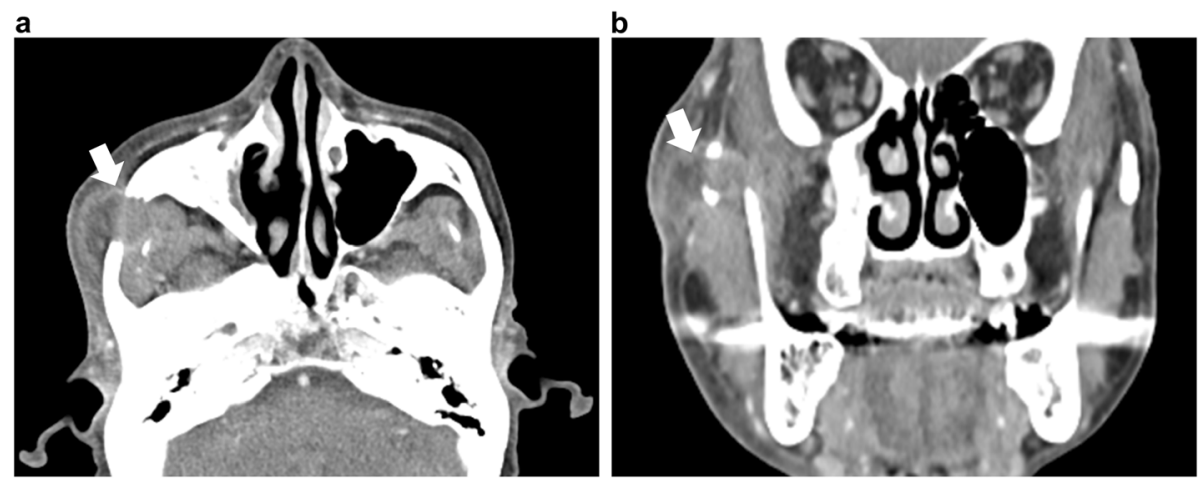

Fig. 2 Pretreatment contrast-enhanced CT (a: axial b: coronal). The lesion (arrow) is located in the right zygomatic arch and has a maximum diameter of $30 \mathrm{~mm}$; bone destruction and surrounding contrast effects are seen. A part of the lesion has extended to the subcutaneous area of the cheek

recurrence (Fig. 5). Inflammatory markers including ESR and CRP remained negative. There were no cosmetic abnormalities or sequelae.

\section{Discussion and conclusions}

Isolated cryptococcal osteomyelitis is a rare entity [2-4]. According to previous reports, including systematic reviews $[5,11]$, the predominant complaints included soft tissue swelling and pain [5]. On the laboratory tests, ESR which reflects bone infection was elevated frequently [5].
Both FNAC and open biopsy were commonly performed and found to be useful for the diagnosis. However, in recent years, FNAC is recommended owing to its minimally invasive nature [5]. Fungal cultures have been reported to show a high positive rate and Cryptococcus neoformans was identified the most frequently as the causative organism [5]. Imaging findings of cryptococcal osteomyelitis have no typical features, and several previous case reports have documented lesions mimicking malignant tumors $[5,11]$. The treatment strategy greatly
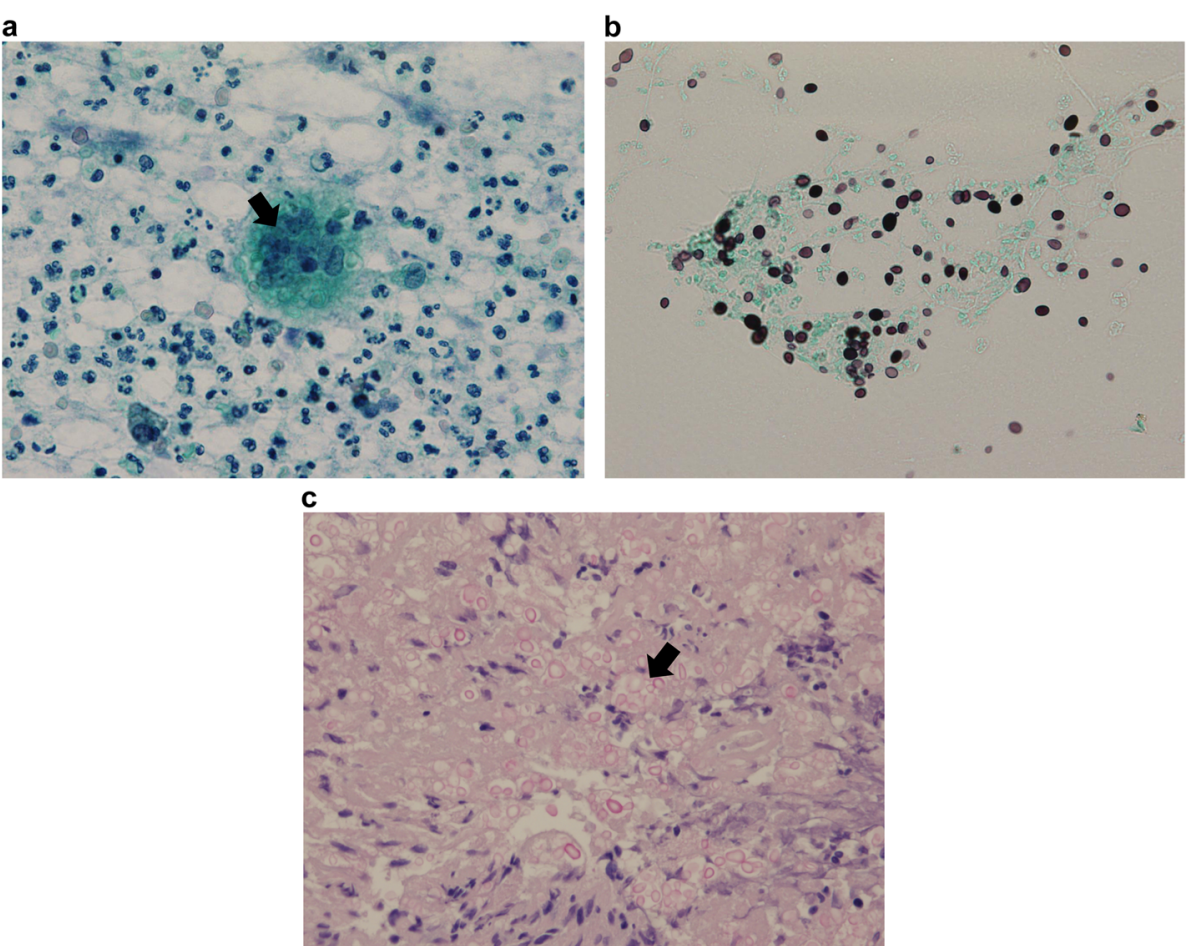

Fig. 3 Histopathological findings. a: Papanicolaou stained smear showing several yeast-like cells phagocytosed by macrophage (arrow) $(\times 400)$. b: Grocott staining yielded positive results $(\times 400)$. c: Mucicarmine staining of biopsy specimen showing numerous cryptococci; yeast-like cells with stained mucopolysaccharide capsules are seen (arrow) $(\times 400)$ 


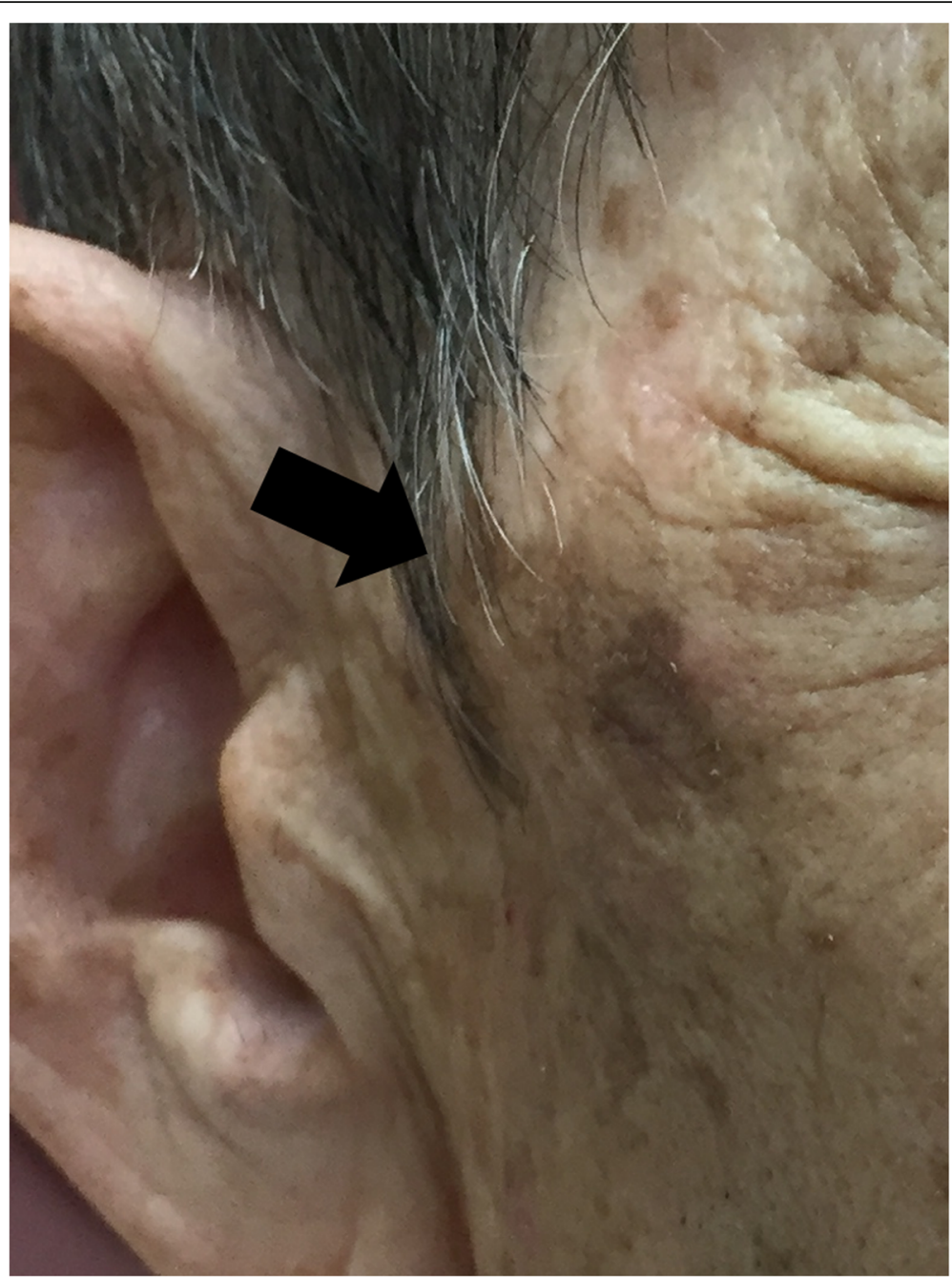

Fig. 4 Photograph of the patient obtained 2 weeks after initiation of treatment. Swelling and erythema have resolved (arrow)
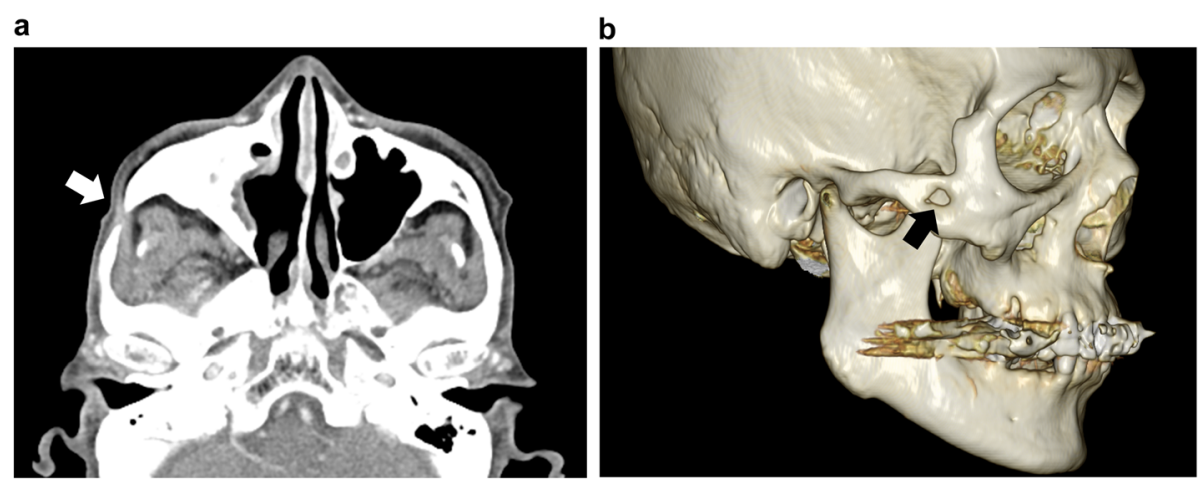

Fig. 5 Contrast-enhanced CT (a: axial; b: three-dimensional imaging) 1 year and 3 months after completion of treatment. The lesion of the zygomatic region has resolved; a partial defect of the zygomatic bone is seen (arrow) 
depends on the absence or presence of disseminated infection. To rule this out, several examinations such as brain MRI, chest CT, LP, tests of serum and CFS cryptococcal antigen titer, and fungal blood and CSF cultures were performed. In addition, a past history, comorbidities such as diabetes, and serology tests for hepatitis virus and HIV are referenced to identify immunodeficiency. Except for the infection of lungs and central nervous system, there are no standardized treatment protocols for cryptococcal infection of specific body sites. A combination of antifungal therapy and surgical debridement has been used to treat many patients with osseous cryptococcosis $[3,5,12,13]$. According to the Infectious Disease Society of America, oral fluconazole ( $400 \mathrm{mg}$ per day for $6-12$ months) is the treatment of choice for immunocompetent patients with non-meningeal, non-pulmonary cryptococcosis $[5,14,15]$. Several case reports have documented successful treatment of patients with isolated cryptococcal osteomyelitis with fluconazole alone due to good oral availability $[3,5,14,16]$. Although the outcomes of disseminated cryptococcosis are typically unfavorable, immunocompetent patients with isolated osteomyelitis have a good prognosis $[5,17]$.

Our patient presented with typical chief complaints and laboratory data of mild elevation of ESR. Owing to the localized inflammation, no systemic signs such as fever or fatigue were observed. We performed both FNAC and a needle biopsy to obtain a definitive diagnosis of this very rare disease. However, as FNAC clearly showed cryptococcal infection in our patient, an invasive needle biopsy may not have been necessary. The culture of the aspirate showed the growth of Cryptococcus neoformans, the most major causative organism.

We couldn't diagnose only by the imaging findings. The main differential diagnosis of trauma, extension of otogenic bacterial infection, and malignant bone tumor were excluded based on the absence of trauma history, CT findings and laboratory data, and histopathological diagnosis, respectively. By the results of several examinations that performed generally, we ruled out disseminated infection and diagnosed our case as isolated osteomyelitis in an immunocompetent patient consequently. However, the serum cryptococcal antigen levels should have been examined, and thereby, additional tests should be performed for cellular and innate immunity, such as that for CD4 lymphopenia, lymphocyte subsets, and serum immunoglobulins, to rule out immunodeficiency caused by factors other than aging.

Since the excision of the zygomatic bone would have caused cosmetic defects, our patient was treated with oral fluconazole alone and was successfully cured. If cryptococcal resistance to fluconazole was observed, another effective antifungal agent would have been administered instead of the surgical treatment. Our patient showed no clinical and radiographical signs of recurrence; however, a palpable partial defect of the zygomatic bone remained. Moreover, there was no gross discrepancy between the two sides of the face on visual examination. The zygomatic bone is a key element of the craniofacial skeleton and plays an important role in maintaining the face shape [18]. Previous reports suggested that it is desirable to reconstruct surgical defects or deformation of the zygomatic bone caused by fracture $[19,20]$. In our patient, the shape of the zygomatic bone was maintained after treatment. However, the presence of a large defect or occurrence of secondary fracture may have caused cosmetic deformities as well as ocular or mandibular dysfunction. In addition, because the masticatory muscles and the temporomandibular joint are in close proximity to this bone, trismus is liable to develop due to the spread of inflammation, as seen in our patient. In case of prolonged and intense inflammation, trismus may not completely resolve after treatment. For these reasons, early diagnosis and treatment of zygomatic lesions are desirable.

Our case may have a risk of secondary fracture or recurrence in the future. For secondary fractures with dysfunction, fixation surgery will be performed. In addition, fluconazole can be re-administered, which has been found effective for recurrence.

In summary, we report the first case of cryptococcal isolated osteomyelitis of the zygomatic bone in an immunocompetent patient without history of trauma. Although the imaging findings were atypical, FNAC, needle biopsy, and fungal culture were useful in obtaining a definitive diagnosis. Patients with isolated osteomyelitis and may be cured with oral fluconazole alone.

\section{Abbreviations \\ ESR: Erythrocyte sedimentation rate; CT: Computed tomography; FNAC: Fine- needle aspiration cytology; HIV: Human immunodeficiency virus; LP: Lumbar puncture; CSF: Cerebrospinal fluid; MRI: Magnetic resonance imaging}

\section{Acknowledgements}

Not applicable.

\section{Authors' contributions \\ TM was responsible for the acquisition and interpretation of the patient data and writing the original draft. SM reviewed and edited the manuscript. TY supervised and reviewed the manuscript. All authors read and approved the final manuscript.}

\section{Authors' information (optional)}

Not applicable.

\section{Funding}

This research did not receive any specific grant from funding agencies in the public, commercial, or not-for-profit sectors.

Availability of data and materials

The main data used or analyzed in this case report are included in this published article. More detailed data are available from the corresponding author on reasonable request. 


\section{Ethics approval and consent to participate}

The need for approval was waived off by the Institutional Ethics Review Board of our institution. Written informed consent of the patient was obtained prior to publication of this case report.

\section{Consent for publication}

Verbal and written consent for publication of this case report and any accompanying images were obtained from the patient.

\section{Competing interests}

The authors declare that they have no competing interests.

Received: 5 March 2020 Accepted: 27 May 2020

Published online: 05 June 2020

\section{References}

1. Kiertiburanakul S, Wirojtananugoon S, Pracharktam R, Sungkanuparph S. Cryptococcosis in human immunodeficiency virus-negative patients. Int J Infect Dis. 2006;10:72-8.

2. Wood L, Miedzinski L. Skeletal cryptococcosis: case report and review of the literature. Can J Infect Dis. 1996;7:125-32.

3. Medaris LA, Ponce B, Hyde Z, Delgado D, Ennis D, Lapidus W, et al. Cryptococcal osteomyelitis: a report of 5 cases and a review of the recent literature. Mycoses. 2016:59:334-42.

4. Al-Tawfiq JA, Ghandour J. Cryptococcus neoformans abscess and osteomyelitis in an immunocompetent patient with tuberculous lymphadenitis. Infection. 2007;35:377-82.

5. Zhou HX, Lu L, Chu T, Wang T, Cao D, Li F, et al. Skeletal cryptococcosis from 1977 to 2013. Front Microbiol. 2014;5:740.

6. Armonda RA, Fleckenstein JM, Brandvold B, Ondra SL. Cryptococcal skull infection: a case report with review of the literature. Neurosurgery. 1993;32: 1034-6 discussion 1036.

7. Italiano A, Yen BC, Rosenthal SA, Rafii M. Cryptococcal osteomyelitis with septic arthritis. Orthopedics. 2001;24:59-60.

8. Sang J, Yang Y, Fan Y, Wang G, Yi J, Fang W, et al. Isolated iliac cryptococcosis in an immunocompetent patient. PLoS Negl Trop Dis. 2018; 12:e0006206.

9. Qin Y, Li TC, Cong TC, Liu YH. Abscess of zygomatic root: A rare otogenic complication. Chin Med J. 2017;130:749-50.

10. Sathe N. Zygomatic abscess as a complication of otitis media. Natl J Maxillofac Surg. 2011;2:181-3.

11. Witte DA, Chen I, Brady J, Ramzy I, Truong LD, Ostrowski ML. Cryptococcal osteomyelitis. Report of a case with aspiration biopsy of a humeral lesion with radiologic features of malignancy. Acta Cytol. 2000:44:815-8.

12. Jain $D$, Najjar M, Azher $Q$, Bachuwa G. Cryptococcal sternal osteomyelitis in a healthy woman: a review of Cryptococcus neoformans. BMJ Case Rep. 2013;2013.

13. Ramkillawan $Y$, Dawood H, Ferreira N. Isolated cryptococcal osteomyelitis in an immune-competent host: a case report. Int J Infect Dis IJID Off Publ Int Soc Infect Dis. 2013;17:e1229-31.

14. Zhou HX, Ning GZ, Feng SQ, Jia HW, Liu Y, Feng HY, et al. Cryptococcosis of lumbar vertebra in a patient with rheumatoid arthritis and scleroderma: case report and literature review. BMC Infect Dis. 2013;13:128.

15. Perfect JR, Dismukes WE, Dromer F, Goldman DL, Graybill JR, Hamill RJ, et al. Clinical practice guidelines for the management of cryptococcal disease: 2010 update by the infectious diseases society of america. Clin Infect Dis. 2010;50:291-322.

16. Ruddy BE, Mayer AP, Ko MG, Labonte HR, Borovansky JA, Boroff ES, et al. Coccidioidomycosis in African Americans. Mayo Clin Proc. 2011;86:63-9.

17. Qadir I, Ali F, Malik UZ, Umer M. Isolated cryptococcal osteomyelitis in an immunocompetent patient. J Infect Dev Ctries. 2011;5:669-73.

18. Gai Z, Yu X, Zhu M. The evolution of the zygomatic bone from agnatha to tetrapoda. Anat Rec (Hoboken, NJ). 2017;300:16-29.

19. Brown JS, Shaw RJ. Reconstruction of the maxilla and midface: introducing a new classification. Lancet Oncol. 2010;11:1001-8.

20. Andrades P, Rosenthal EL, Carroll WR, Baranano CF, Peters GE. Zygomaticmaxillary buttress reconstruction of midface defects with the osteocutaneous radial forearm free flap. Head Neck. 2008;30:1295-302.

\section{Publisher's Note}

Springer Nature remains neutral with regard to jurisdictional claims in published maps and institutional affiliations.

Ready to submit your research? Choose BMC and benefit from:

- fast, convenient online submission

- thorough peer review by experienced researchers in your field

- rapid publication on acceptance

- support for research data, including large and complex data types

- gold Open Access which fosters wider collaboration and increased citations

- maximum visibility for your research: over $100 \mathrm{M}$ website views per year

At $\mathrm{BMC}$, research is always in progress.

Learn more biomedcentral.com/submissions 